\title{
Visco-Elastic Unsteady Mhd Flow Between Two Horizontal Parallel Plates With Hall Current
}

\author{
Rita Choudhury ${ }^{1}$ and Sajal Kumar Das ${ }^{2}$ \\ ${ }^{1}$ Department of Mathematics, Gauhati University, Guwahati-781 014, Assam, India \\ ${ }^{2}$ Department of Mathematics, Bajali College, Pathsala,Barpeta Assam, India.
}

\begin{abstract}
An analysis of unsteady MHD flow of an electrically conducting visco-elastic fluid confined between two horizontal parallel non conducting plates in presence of a transverse magnetic field and Hall current is presented. The lower plate is a stretching sheet while the upper one is an oscillating porous plate, which is oscillating in its own plane. The motion of the fluid is produced by the stretching of the lower plate. A constant suction is applied at the upper plate and the stretching velocity is taken to be a linear function of distance along the channel. The equations governing the flow field are solved by perturbation technique. Expressions for velocity distribution of the flow field and non-dimensional skin-friction coefficient are obtained and presented graphically to observe the visco-elastic effect in combination of other flow parameters involved in the solution. The flow field is observed to be considerably affected by the visco-elastic parameter.
\end{abstract}

Keywords - Hall current, MHD, Oscillating porous plate, Perturbation technique, Stretching sheet, Suction parameter, Visco-elastic.

\section{Introduction}

Analysis of flow over a stretching sheet attracts the interest of many researchers in a large scale because of its application in different areas such as in the manufacture of sheeting material through an extrusion process, the cooling of bath, the boundary layer along material handling conveyers, the aerodynamic extrusion of plastic sheets, glass and polymer industries, fiber industry etc. Recently, the boundary layer flow over a moving continuous solid surface has become inevitable in various fields of engineering processes. Borkakoti and Bharali [1] have studied the MHD flow of a conducting fluid with heat transfer between two horizontal parallel plates where the lower one is stretching and the upper one is a porous solid plate. Chiam [2] has analyzed the motion of micro polar fluids over stretching sheet. Rajagopal et al. [3] have studied the flow of a visco-elastic fluid over a stretching sheet. Agarwal et al. [4] have obtained the solution of flow and heat transfer of a micro polar fluid over a stretching sheet using finite element technique. The visco-elastic MHD flow past a stretching sheet has been studied by Anderson [5]. He has compared the effect of an external magnetic field and the visco-elastic effect on the flow. Anderson et al. [6] has studied the motion of power law fluid over a stretching sheet. They have investigated the flow of an electrically conducting visco-elastic fluid past a flat and a impermeable elastic sheet. Char [7] has extended this work to study heat as well as mass transfer. The coupled stretching flow of a two dimensional viscous incompressible fluid through a channel bounded by naturally permeable bed has been studied by Chauhan [8]. The unsteady flow over a stretching surface with a magnetic field in a rotating fluid has been studied by Takhar and Nath [9]. Kumari et al. [10] have obtained the analytical solution of the boundary layer equations over a stretching sheet with mass transfer using series method. Sharma and Mishra [11] have investigated the steady MHD flow through horizontal channel. A numerical solution for power law velocity distribution of stretching plate has been obtained by Phukan [12]. Bhardwaj [13] has analyzed the steady two dimensional flow of viscous, incompressible fluid through a channel bounded by a plane stretched sheet and naturally permeable bed. Lodha and Tak [14] have analyzed the boundary layer flow of a hydromagnetic fluid over a stretching sheet in the presence of uniform transverse magnetic field with heat transfer taking dissipation function into account. The important fact is that the influence of Hall current effects was not concentrated much in all the above studies. If a conductor or a semi conductor has current flowing in it because of an applied electric field and a transverse magnetic field, there develops a component of electric field in the direction orthogonal to both the applied electric field and magnetic field, resulting in a voltage difference between the sides of the conductor. This phenomenon is known as the Hall Effect. In an ionized gas, when the strength of the magnetic field is large one cannot neglect the effect of Hall current. These Hall currents are particularly important as they produce considerable changes in the flow pattern, when the magnetic field is considerably large. Gutpa [15] has studied the Hall current effects in the steady MHD flow of an electrically conducting fluid past an infinite porous flat plate. Kumar et al. [16] have analyzed the thermal instability of visco-elastic fluid permeated with suspended particles in hydromagnetics in porous medium. Kumar and Singh [17] have studied the instability of two rotating visco-elastic (Walters B') superposed fluids with suspended particles in porous medium. The laminar fully developed mixed convection with viscous dissipation in a uniformly heated vertical 
Visco-Elastic Unsteady Mhd Flow Between Two Horizontal Parallel Plates With Hall Current

double-passage channel has been studied by Mostafa and Salah [18]. Bakier and Mansour [19] have studied the combination of magnetic field and thermophoresis particle deposition in free convection boundary layer from a vertical flat plate embedded in a porous medium. Abdallah [20] has investigated analytic solution of heat and mass transfer over a permeable stretching plate affected by chemical reaction, internal heating, Dufour-Soret effect, and Hall Effect. Raju et al. [21] have studied the Hall-current effects on unsteady MHD flow between stretching sheet and an oscillating porous upper parallel plate with constant suction.

The object of the present author is to study the unsteady MHD flow of an electrically conducting viscoelastic fluid characterized by Walters liquid (Model B') confined between two horizontal parallel non conducting plates in presence of a transverse magnetic field and the effects of Hall current where the lower plate is a stretching sheet and the upper one is an oscillating porous plate with constant suction, which is oscillating in its own plane and to observe the visco-elastic effects on flow field along with other flow parameters. Here the motion of the fluid is produced by the stretching of the lower plate and suction at the upper porous plate.

The constitutive equation for Walters liquid (Model B') is

$\sigma^{i k}=-p g_{i k}+2 \eta_{0} e^{i k}-2 K_{0} e^{i k}$

where $\sigma^{i k}$ is the stress tensor, $p$ is isotropic pressure, $g_{i k}$ is the metric tensor of a fixed co-ordinate system $x^{i}, v^{i}$ is the velocity vector, the contravariant form of $e^{i k}$ is given by

$e^{i k}=\frac{\partial e^{i k}}{\partial t}+v^{m} e^{i k}{ }_{m}-v^{i}{ }_{m} e^{i m}-v^{i}{ }_{m} e^{m k}$

It is the convected derivative of the deformation rate tensor $\mathrm{e}^{\mathrm{ik}}$ defined by

$2 \mathrm{e}^{\mathrm{ik}}=\mathrm{v}^{\mathrm{i}},{ }_{\mathrm{k}}+\mathrm{v}^{\mathrm{k}},{ }_{\mathrm{i}}$

Here $\eta_{0}$ is the limiting viscosity at the small rate of shear which is given by

$\eta_{0}=\int_{0}^{\infty} N(\tau) d \tau$ and $k_{0}=\int_{0}^{\infty} \tau N(\tau) d \tau$

$\mathrm{N}(\tau)$ being the relaxation spectrum as introduced by Walters $[22,23]$. This idealized model is a valid approximation of Walters liquid (Model B') taking very short memories into account so that terms involving $\int_{0}^{\infty} \tau^{n} N(\tau) d \tau, \quad n \geq 2$

have been neglected.

\section{Mathematical Formulation}

The unsteady MHD flow of an electrically conducting visco-elastic fluid characterized by Walters liquid (Model $\mathrm{B}^{\prime}$ ) confined between two horizontal parallel non conducting plates in presence of a transverse magnetic field and the effects of Hall current where the lower plate is a stretching sheet and the upper one is an oscillating porous plate with constant suction, which is oscillating in its own plane is considered. The $x$-axis be taken along the lower stretching sheet in the flow direction and $y$-axis is taken perpendicular to the sheet. Two equal and opposite forces are introduced along the $x$-axis to stretch the lower plate so that the position of the origin remains unaltered. The fluid is sucked through the upper porous plate with constant velocity $V_{0}$. A transverse magnetic field $B_{0}$ of small magnitude is applied so that the induced magnetic field is negligible in comparison with applied magnetic field.

\section{The governing equations:}

\subsection{Equation of continuity:}

$\frac{\partial \bar{u}}{\partial \bar{x}}+\frac{\partial \bar{v}}{\partial \bar{y}}=0$

\subsection{Equations of momentum:}

$$
\begin{aligned}
& \frac{\partial \bar{u}}{\partial \bar{t}}+\bar{u} \frac{\partial \bar{u}}{\partial \bar{x}}+\bar{v} \frac{\partial \bar{u}}{\partial \bar{y}}=-\frac{1}{\rho} \frac{\partial \bar{p}}{\partial \bar{x}}+v\left(\frac{\partial^{2} \bar{u}}{\partial \bar{x}^{2}}+\frac{\partial^{2} \bar{u}}{\partial \bar{y}^{2}}\right)-\frac{K_{0}}{\rho}\left(\frac{\partial^{s} \bar{u}}{\partial \bar{t} \partial \bar{x}^{-2}}+\frac{\partial^{s} \bar{u}}{\partial \bar{t} \partial \bar{y}^{2}}+\bar{u} \frac{\partial^{s} \bar{u}}{\partial \bar{x}^{s}}+\bar{u} \frac{\partial^{s} \bar{u}}{\partial \bar{x}^{2} \partial \bar{y}^{2}}+\bar{v} \frac{\partial^{s} \bar{u}}{\partial \bar{y} \partial \bar{x}^{-2}}+\bar{v} \frac{\partial^{s} \bar{u}}{\partial \bar{y}^{s}}-\right. \\
& \left.3 \frac{\partial \bar{u}}{\partial \bar{x}} \frac{\partial^{2} \bar{u}}{\partial \bar{x}^{2}}-\frac{\partial \bar{v}}{\partial \bar{y}} \frac{\partial^{2} \bar{u}}{\partial \bar{y}^{2}}-\frac{\partial \bar{u}}{\partial \bar{y}} \frac{\partial^{2} \bar{v}}{\partial \bar{x}^{2}}-\frac{\partial \bar{u}}{\partial \bar{y}} \frac{\partial^{2} \bar{u}}{\partial \bar{x} \partial \bar{y}}-2 \frac{\partial \bar{v}}{\partial \bar{x}} \frac{\partial^{2} \bar{u}}{\partial \bar{x} \partial \bar{y}}\right) \\
& \frac{\partial \bar{v}}{\partial \bar{t}}+\bar{u} \frac{\partial \bar{v}}{\partial \bar{x}}+\bar{v} \frac{\partial \bar{v}}{\partial \bar{y}}=-\frac{1}{\rho} \frac{\partial \bar{p}}{\partial \bar{y}}+v\left(\frac{\partial^{2} \bar{v}}{\partial \bar{x}^{2}}+\frac{\partial^{2} \bar{v}}{\partial \bar{y}^{2}}\right)-\frac{K_{0}}{\rho}\left(\frac{\partial^{8} \bar{v}}{\partial \bar{t} \theta \bar{x}^{2}}+\frac{\theta^{8} \bar{v}}{\partial \bar{t} \theta \bar{y}^{2}}+\bar{u} \frac{\partial^{8} \bar{v}}{\partial \bar{x}^{8}}+\bar{u} \frac{\partial^{8} \bar{v}}{\partial \bar{x} \theta \bar{y}^{2}}+\bar{v} \frac{\partial^{8} \bar{v}}{\partial \bar{y} \theta \bar{x}^{2}}+\bar{v} \frac{\partial^{8} \bar{v}}{\partial \bar{y}^{3}}-\frac{\partial \bar{u}}{\partial \bar{x}} \frac{\partial^{2} \bar{v}}{\partial \bar{x}^{2}}-\right. \\
& \left.\frac{\partial \bar{v}}{\partial \bar{x}} \frac{\partial^{2} \bar{u}}{\partial \bar{y}^{2}}-\frac{\partial \bar{v}}{\partial \bar{x}} \frac{\partial^{2} \bar{v}}{\partial \bar{x} \theta \bar{y}}-3 \frac{\partial \bar{v}}{\partial \bar{y}} \frac{\partial^{2} \bar{v}}{\partial \bar{y}^{2}}-2 \frac{\partial \bar{u}}{\partial \bar{y}} \frac{\partial^{2} \bar{v}}{\partial \bar{x} \theta \bar{y}}\right)-\frac{\sigma_{\theta_{B} B_{0}{ }^{2}}}{\rho\left(1+m^{2}\right)} \bar{u}
\end{aligned}
$$

Subject to boundary conditions:

$\bar{y}=0: \bar{u}=c \bar{x}, \quad \bar{v}=0$

$\bar{y}=h: \quad \bar{u}=\bar{U}_{0}\left(1+\epsilon e^{i \bar{\omega} \bar{t}}\right), \bar{v}=V_{0}$

We introduce the non-dimensional quantities 
$x=\frac{\bar{x}}{h}, y=\frac{\bar{y}}{h}, \bar{t}=\frac{t}{c}, \bar{\omega}=\omega c, R_{e}=\frac{c h^{2}}{v}, M=B_{0} h \sqrt{\frac{\sigma_{e}}{\rho v}}, M_{1}=\frac{M}{\sqrt{1+m^{2}}}, U_{0}=\frac{\bar{U}_{0}}{h}$

such that the equation (6) admits the self similar solution

$\bar{u}=c \bar{x} f^{\prime}(y, t), \bar{v}=-\operatorname{ch} f(y, t)$

where dash denotes differentiation with respect to $\mathrm{y}$.

Using (10) and (11) in (7) and (8) we get

$\frac{\partial f^{\prime}}{\partial t}+f^{2}-f f^{\prime \prime}-\frac{1}{R_{e}} f^{\prime \prime \prime}+K_{1}\left(\frac{\partial f^{\prime \prime \prime}}{\partial t}+2 f^{\prime} f^{\prime \prime \prime}-f f^{i v}-f^{\prime \prime 2}\right)+\frac{M_{1}{ }^{2}}{R_{e}} f^{\prime}=-\frac{1}{\rho x c^{2} h^{2}} \frac{\partial \bar{P}}{\partial x}$

$\frac{\partial f}{\partial t}-f f^{\prime}-\frac{1}{R_{e}} f^{\prime \prime}+K_{1}\left(\frac{\partial f^{\prime \prime}}{\partial t}-f f^{\prime \prime \prime}+3 f^{\prime} f^{\prime \prime}\right)=\frac{1}{\rho c^{2} h^{2}} \frac{\partial \bar{P}}{\partial y}$

where $K_{1}=\frac{K_{0}}{\rho h^{2}}, B_{0}$ is uniform transverse magnetic field, $c$ is rate of stretching, $h$ is width of the channel, $p$ is pressure, $\mathrm{R}_{\mathrm{e}}$ is stretching Reynolds number, $t$ is time, $U_{0}$ is characteristic velocity of the upper plate, $V_{0}$ is suction velocity through upper plate, $y$ is similarity variable, $v$ is kinematic viscosity, $\rho$ is density of the fluid , $\sigma_{e}$ is electric conductivity, $\omega$ is frequency.

Differentiating (13) with respect to $x$, we get

$\frac{\partial^{2} \bar{P}}{\partial x \partial y}=0$

From equation (12) and (14), we have

$f^{\prime \prime \prime}+R_{e} f f^{\prime \prime}-R_{e} \frac{\partial f^{\prime}}{\partial t}-R_{e} f^{\prime^{2}}-K_{1} R_{e}\left(\frac{\partial f^{\prime \prime \prime}}{\partial t}+2 f^{\prime} f^{\prime \prime \prime}-f f^{i v}-f^{\prime \prime 2}\right)-M_{1}{ }^{2} f^{\prime}=C(t)$

where $C(t)$ is constant with respect to $x$ and $y$.

The corresponding boundary conditions are:

$y=0: f=0, f^{\prime}=1$

$y=1: f=-\beta, f^{\prime}=\alpha\left(1+\epsilon e^{i \omega t}\right)$

where $\alpha=\frac{U_{0}}{c x}, \beta=\frac{V_{0}}{c h}$

\section{Method of solution:}

Keeping in view the boundary conditions, we assume for $\varepsilon \ll 1$,

$f(y, t)=f_{0}(y)+\varepsilon e^{i \omega t} f_{1}(y)$

$C(t)=C_{0}+\varepsilon e^{i \omega t} C_{1}$

Using (17) in (15) and separating steady and unsteady parts we get,

$f_{0}^{\prime \prime \prime}+R_{e} f_{0} f_{0}^{\prime \prime}-R_{e} f_{0}{ }^{2}-M_{1}^{2} f_{0}^{\prime}-K_{1} R_{e}\left(2 f_{0}^{\prime} f_{0}^{\prime \prime \prime}-f_{0} f_{0}{ }^{i v}-f_{0}{ }^{\prime 2}\right)=C_{0}$

$f_{1}^{\prime \prime \prime}+R_{e} f_{1} f_{0}^{\prime \prime}+R_{e} f_{0} f_{1}^{\prime \prime}-2 R_{e} f_{0}^{\prime} f_{1}^{\prime}-M_{1}^{2} f_{1}^{\prime}-i \omega R_{e} f_{1}^{\prime}-K_{1} R_{e}\left(2 f_{0}^{\prime} f_{1}^{\prime \prime \prime}+2 f_{0}^{\prime \prime \prime} f_{1}^{\prime}-f_{0} f_{1}^{i v}-f_{1} f_{0}{ }^{i v}-\right.$

$\left.2 f_{0}^{\prime \prime} f_{1}^{\prime \prime}+i \omega f_{1}^{\prime \prime \prime}\right)=C_{1}$

with modified boundary conditions:

$y=0: f_{0}=0, f_{1}=0, f_{0}^{\prime}=1, f_{1}^{\prime}=1 \quad$ at $\mathrm{y}=0$

$y=1: f_{0}=-\beta, f_{1}=0, f_{0}^{\prime}=\alpha, f_{1}^{\prime}=\alpha$ at $\mathrm{y}=1$

For $R_{e} \ll 1$, we now apply the perturbation scheme

$f_{0}=f_{00}+R_{e} f_{01}+o\left(R_{e}{ }^{2}\right)$

$f_{1}=f_{10}+R_{e} f_{11}+o\left(R_{e}^{2}\right)$

$C_{0}=C_{00}+R_{e} C_{01}+o\left(R_{e}^{2}\right)$

$C_{1}=C_{10}+R_{e} C_{11}+o\left(R_{e}{ }^{2}\right)$

Substituting (21) in (18) and (19) and comparing the coefficients of various powers of $R_{e}$ we get the following sets of equations:

\subsection{Zeroth order equations:}

$f_{00}^{\prime \prime \prime}-M_{1}^{2} f_{00}^{\prime}=C_{00}$

$f_{01}^{\prime \prime \prime}+f_{00} f_{00}^{\prime \prime}-f_{00}{ }^{\prime 2}-M_{1}{ }^{2} f_{01}^{\prime}-K_{1}\left(2 f_{00}{ }^{\prime \prime \prime \prime} f_{00}-f_{00} f_{00}{ }^{i v}-f_{00}{ }^{\prime \prime 2}\right)=C_{01}$

with relevant boundary conditions:

$y=0: f_{00}=0, f_{01}=0, f_{00}{ }^{\prime}=1, f_{01}{ }^{\prime}=0$

$y=1: \quad f_{00}=-\beta, f_{01}=0, f_{00}{ }^{\prime}=\alpha, f_{01}{ }^{\prime}=0$

\subsection{First order equations:}

$f_{10}^{\prime \prime \prime}-M_{1}^{2} f_{10}^{\prime}=C_{10}$ 
$f_{11}{ }^{\prime \prime \prime}+f_{10} f_{00}{ }^{\prime \prime}+f_{00} f_{10}{ }^{\prime \prime}-2 f_{00}{ }^{\prime} f_{10}{ }^{\prime}-M_{1}{ }^{2} f_{11}{ }^{\prime}-i \omega f_{10}{ }^{\prime}-K_{1}\left(2 f_{00}{ }^{\prime} f_{10}{ }^{\prime \prime \prime}+2 f_{00}{ }^{\prime \prime \prime} f_{10}{ }^{\prime}-f_{00} f_{10}{ }^{i v}-f_{10} f_{00}{ }^{i v}-\right.$ $\left.2 f_{00} " f_{10} "+i \omega f_{10}^{\prime \prime \prime}\right)=C_{11}$

Subject to boundary conditions:

$y=0: \quad f_{10}=0, f_{11}=0, f_{10}^{\prime}=0, f_{11}^{\prime}=0$

$y=1: \quad f_{10}=0, f_{11}=0, f_{10}^{\prime}=\alpha, f_{11}^{\prime}=0$

On solving equations (22), (23), (25) and (26), we obtain,

$f_{00}=B_{1}+B_{2} e^{M_{1} y}+B_{3} e^{-M_{1} y}-\frac{C_{00}}{M_{1}{ }^{2}} y$

$f_{01}=B_{4}+B_{5} e^{M_{1} y}+B_{6} e^{-M_{1} y}-\frac{b_{1}}{M_{1}{ }^{2}} y+\frac{b_{2}}{2 M_{1}{ }^{2}} y e^{M_{1} y}+\frac{b_{3}}{2 M_{1}{ }^{2}} y e^{-M_{1} y}+\frac{b_{4}}{2 M_{1}{ }^{2}} e^{M_{1} y}\left(\frac{y^{2}}{2}-\frac{3 y}{2 M_{1}}\right)$

$+\frac{b_{5}}{2 M_{1}{ }^{2}} e^{-M_{1} y}\left(\frac{y^{2}}{2}+\frac{3 y}{2 M_{1}}\right)-\frac{C_{01}}{M_{1}{ }^{2}} y$

$f_{10}=B_{7}+B_{8} e^{M_{1} y}+B_{9} e^{-M_{1} y}-\frac{C_{10}}{M_{1}^{2}} y$

$f_{11}=B_{10}+B_{11} e^{M_{1} y}+B_{12} e^{-M_{1} y}-\frac{b_{9}}{M_{1}{ }^{2}} y+\frac{b_{10}}{2 M_{1}{ }^{2}} y e^{M_{1} y}+\frac{b_{11}}{2 M_{1}{ }^{2}} y e^{-M_{1} y}+\frac{b_{12}}{2 M_{1}{ }^{2}} e^{M_{1} y}\left(\frac{y^{2}}{2}-\frac{3 y}{2 M_{1}}\right)+$ $\frac{b_{13}}{2 M_{1}{ }^{2}} e^{-M_{1} y}\left(\frac{y^{2}}{2}+\frac{3 y}{2 M_{1}}\right)-\frac{C_{11}}{M_{1}{ }^{2}} y$

$f_{00}{ }^{\prime}=B_{2} M_{1} e^{M_{1} y}-B_{3} M_{1} e^{-M_{1} y}-\frac{C_{00}}{M_{1}{ }^{2}}$

$f_{01}^{\prime}=B_{5} M_{1} e^{M_{1} y}-B_{6} M_{1} e^{-M_{1} y}-\frac{M_{1}{ }^{2}}{M_{1}{ }^{2}}+\frac{v_{2}}{2 M_{1}{ }^{2}}\left(e^{M_{1} y}+M_{1} y e^{M_{1} y}\right)+\frac{v_{8}}{2 M_{1}{ }^{2}}+\frac{v_{4}}{2 M_{1}{ }^{2}}\left\{M_{1} e^{M_{1} y}\left(\frac{y}{2}-\frac{s y}{2 M_{1}}\right)+\right.$

$$
\left.e^{M_{1} y}\left(y-\frac{3}{2 M_{1}}\right)\right\}+\frac{b_{5}}{2 M_{1}^{2}}\left\{-M_{1} e^{-M_{1} y}\left(\frac{y^{2}}{2}+\frac{3 y}{2 M_{1}}\right)+e^{-M_{1} y}\left(y+\frac{3}{2 M_{1}}\right)\right\}-\frac{c_{01}}{M_{1}^{2}}
$$

$f_{10}^{\prime}=B_{8} M_{1} e^{M_{1} y}-B_{9} M_{1} e^{-M_{1} y}-\frac{C_{10}}{M_{1}{ }^{2}}$

$f_{11}^{\prime}=B_{11} M_{1} e^{M_{1} y}-B_{12} M_{1} e^{-M_{1} y}-\frac{b_{9}}{M_{1}{ }^{2}}+\frac{b_{10}}{2 M_{1}{ }^{2}}\left(e^{M_{1} y}+M_{1} y e^{M_{1} y}\right)+\frac{b_{11}}{2 M_{1}{ }^{2}}\left(e^{-M_{1} y}-M_{1} y e^{-M_{1} y}\right)+$ $\frac{b_{12}}{2 M_{1}{ }^{2}}\left\{M_{1} e^{M_{1} y}\left(\frac{y^{2}}{2}-\frac{3 y}{2 M_{1}}\right)+e^{M_{1} y}\left(y-\frac{3}{2 M_{1}}\right)\right\}+\frac{b_{13}}{2 M_{1}{ }^{2}}\left\{-M_{1} e^{-M_{1} y}\left(\frac{y^{2}}{2}+\frac{3 y}{2 M_{1}}\right)+e^{-M_{1} y}\left(y+\frac{3}{2 M_{1}}\right)\right\}-\frac{C_{11}}{M_{1}{ }^{2}}$

Finally using (17) and (21) the velocity field is obtained in terms of $f$ as

$f(y, t)=f_{00}(y)+R_{e} f_{01}(y)+\epsilon e^{i \omega t}\left(f_{10}(y)+R_{e} f_{11}(y)\right)$

\subsection{Skin friction:}

The skin friction at the plate $\mathrm{y}=0$ is given by

$\sigma_{0}=\left(\sigma_{x y}\right)_{y=0}=\left[\frac{1}{R e} f^{\prime \prime}(y, t)-K_{1}\left\{\frac{\partial f^{\prime \prime}(y, t)}{\partial t}-3 f^{\prime}(y, t) f^{\prime \prime}(y, t)-f(y, t) f^{\prime \prime \prime}(y, t)\right\}\right]_{y=0}$

The skin friction at the plate $\mathrm{y}=1$ is given by

$\sigma_{1}=\left(\sigma_{x y}\right)_{y=1}=\left[\frac{1}{R e} f^{\prime \prime}(y, t)-K_{1}\left\{\frac{\partial f^{\prime \prime}(y, t)}{\partial t}-3 f^{\prime}(y, t) f^{\prime \prime}(y, t)-f(y, t) f^{\prime \prime \prime}(y, t)\right\}\right]_{y=1}$

The constants are obtained but not given here due to brevity.

\section{Discussion}

The flow field along the $y$-direction at the stretching sheet is characterized by $-\mathrm{f}(y, t)$ and has been illustrated in Fig.s 1 to 3 for the fixed values of the flow parameters $R_{e}=.5, \omega=5, \omega t=\pi / 4, \varepsilon=.01, \alpha=.8, \beta=.8, M=2$ and $M=4$ with variations in $\mathrm{m}$. Here $\alpha$ is the velocity parameter of the upper oscillating porous plate, $\beta$ is the suction parameter, $\mathrm{M}$ is the magnetic parameter and $\mathrm{m}$ is the Hall current parameter. In all these figures, the visco-elastic effect is exhibited by the parameter $K_{1}\left(K_{1}=0,0.15,0.3\right)$. The value 0 of $K_{1}$ corresponds to Newtonian fluid. In numerical calculations, the real part is implied throughout.

Fig.s 1 to 3 illustrate the flow field along $\mathrm{y}$-direction for $\mathrm{m}=0,2$ and 4 respectively for $\mathrm{M}=2$ and 4 with other flow parameters remaining unaltered.

It is noticed from Fig. $1(\mathrm{~m}=0)$ that the velocity profile $v$ in the $y$-direction diminishes with the growth of the visco-elastic parameter $\mathrm{K}_{1}$ in comparision with that of Newtonian fluid for $\mathrm{M}=2$ and $\mathrm{M}=4$ both. It is also observed that the magnitude of $v$ decreases up to $\mathrm{y}=.1$, then increases throughout the centre of the channel up to $\mathrm{y}=.85$ and finally decreases near the moving sheet $(y=1)$. It is further found that the visco-elastic effect is the highest in the centre of the channel with a higher variation for $\mathrm{M}=4$ than for $\mathrm{M}=2$ while it is almost nil near the sheets. It is also seen that the magnitude of $v$ decreases with the increase of $\mathrm{M}$ in the centre of the channel and remains the same near the sheets.

Fig. $2(m=2)$ illustrates that the velocity profile enhances for $M=2$ and $M=4$ with the rising effect of the visco-elastic parameter $\mathrm{K}_{1}$. For $\mathrm{m}=2$ (Fig. 2), the visco-elastic effect is the highest in the centre of the channel with a higher variation for $\mathrm{M}=2$ than for $\mathrm{M}=4$ while it is almost nil near the sheets. It is also observed that the magnitude of $v$ decreases up to $\mathrm{y}=.05$, then increases throughout the centre of the channel up to $\mathrm{y}=.64$ and finally decreases near the moving sheet $(y=1)$ for $M=2$ while the magnitude decreases up to $y=.1$, then increases 
Visco-Elastic Unsteady Mhd Flow Between Two Horizontal Parallel Plates With Hall Current

throughout the centre of the channel up to $y=.84$ and finally decreases near the moving sheet $(y=1)$ for $M=4$. It is further observed that the magnitude of $v$ decreases with the increase of $\mathrm{M}$ in the centre of the channel and remains the same near the sheets. The visco-elastic effect is more prominent for $\mathrm{M}=2$ than for $\mathrm{M}=4$.

Fig. $3(m=4)$ illustrates that the velocity profile enhances for $M=2$ and $M=4$ with the rising effect of the visco-elastic parameter $\mathrm{K}_{1}$. For $\mathrm{m}=4$ (Fig. 3), the visco-elastic effect is the highest in the centre of the channel with a higher variation for $\mathrm{M}=2$ than for $\mathrm{M}=4$ while it is almost nil near the sheets. It is also observed that the magnitude of $v$ increases up to $\mathrm{y}=.55$ and then decreases for both $\mathrm{M}=2$ and $\mathrm{M}=4$. It is also observed that the magnitude of $v$ decreases with the increase of $\mathrm{M}$ in the centre of the channel and remains the same near the sheets.

Fig.s 4 to 6 illustrate the velocity of the flow field along the stretching sheet i.e. along $x$ - direction which is characterized by $\mathrm{f}^{\prime}(y, t)$ for the fixed values of the flow parameters $\mathrm{R}_{\mathrm{e}}=.5, \omega=5, \omega \mathrm{t}=\pi / 4, \varepsilon=.01, \alpha=.8$, $\beta=.8, \mathrm{M}=2$ and $\mathrm{M}=4$ with variations in $\mathrm{m}(=0,2$ and 4$)$.

Fig. $4(\mathrm{~m}=0)$ explains that $\mathrm{f}^{\prime}(y, t)$ and hence the velocity profile $u$ along the stretching sheet i.e. along $x$ direction has an accelerating trend near the stretching sheet and a decelerating trend near the moving sheet with the growth of the visco-elastic parameter $\mathrm{K}_{1}$ for both $\mathrm{M}=2$ and $\mathrm{M}=4$. The magnitude of $u$ decreases near the stretching sheet up to $\mathrm{y}=.4$ and then increases up to the moving sheet for $\mathrm{M}=2$ and the magnitude of $u$ decreases near the stretching sheet up to $y=.5$ and then increases up to the moving sheet for $M=4$. The visco-elastic effect is more prominent for $\mathrm{M}=4$ than for $\mathrm{M}=2$.

Fig. $5(\mathrm{~m}=2)$ illustrates that the velocity profile $u$ diminishes near the stretching sheet and enhances near the moving sheet with the growth of the visco-elastic parameter $K_{1}$ for both $M=2$ and $M=4$. The magnitude of $u$ decreases near the stretching sheet up to $y=.3$, then increases up to $y=.85$ and finally decreases near the moving sheet for $M=2$ while it decreases up to $y=.4$ and then increases up to the moving sheet for $M=4$. The visco-elastic effect is more prominent for $\mathrm{M}=2$ than for $\mathrm{M}=4$.

Fig. $6(\mathrm{~m}=4)$ describes that the velocity profile $u$ declines near the stretching sheet and enhances near the moving sheet with the rising effect of the visco-elastic parameter $K_{1}$ for both $M=2$ and $M=4$. The magnitude of $u$ decreases near the stretching sheet up to $\mathrm{y}=.25$, then increases up to $y=.82$ and finally decreases near the moving sheet for both $M=2$ and $M=4$. The visco-elastic effect is more prominent for $M=2$ than for $M=4$.

Fig.s 7 to 10 illustrate the behavior of skin-friction coefficient against $\alpha$ and $\beta$ on the stretching $(y=0)$ and moving $(y=1)$ sheets respectively for $\mathrm{m}=2, \mathrm{R}_{\mathrm{e}}=.5, \omega=5, \omega \mathrm{t}=\pi / 4, \varepsilon=.01, \mathrm{M}=2$ and $\mathrm{M}=4$.

It is observed from Fig. 7 and Fig. 9 that the skin-friction coefficient $\sigma_{0}$ against $\alpha$ and $\beta$ respectively at the stretching sheet ( $\mathrm{y}=0$ ) diminishes for both $\mathrm{M}=2$ and $\mathrm{M}=4$ with the rise of the visco-elastic parameter $\mathrm{K}_{1}$ in comparision with that of the Newtonian fluid flow $\left(\mathrm{K}_{1}=0\right)$.

Fig.s 8 and Fig. 10 depict that the skin-friction coefficient $\sigma_{1}$ against $\alpha$ and $\beta$ respectively at the moving sheet $(\mathrm{y}=1)$ enhances for both $\mathrm{M}=2$ and $\mathrm{M}=4$ with the growth of the visco-elastic parameter $\mathrm{K}_{1}$ in comparision with that of the Newtonian fluid flow $\left(\mathrm{K}_{1}=0\right)$.

It is also seen from the Fig.s 7 to 10 that the visco-elastic effect is more prominent for $\mathrm{M}=2$ than for $\mathrm{M}=4$.

\section{Figures:}

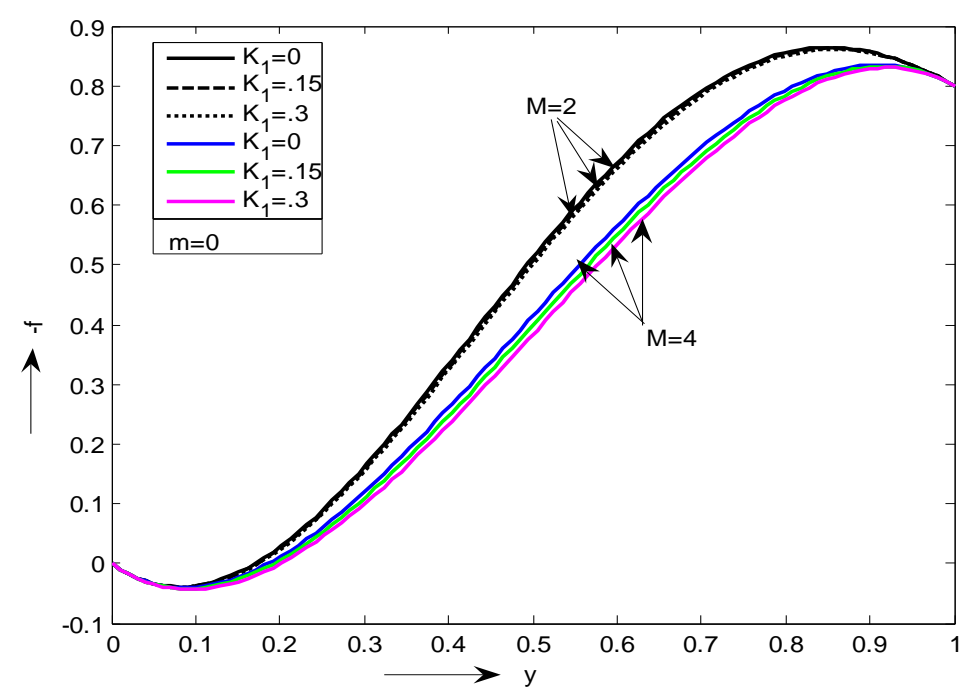

Figure 1: -f against y for $\mathrm{M}=2, \mathrm{M}=4, \mathrm{~m}=0, \alpha=.8, \beta=.8, \mathrm{R}_{\mathrm{e}}=.5, \omega \mathrm{t}=\pi / 4, \omega=5, \varepsilon=.01$ 


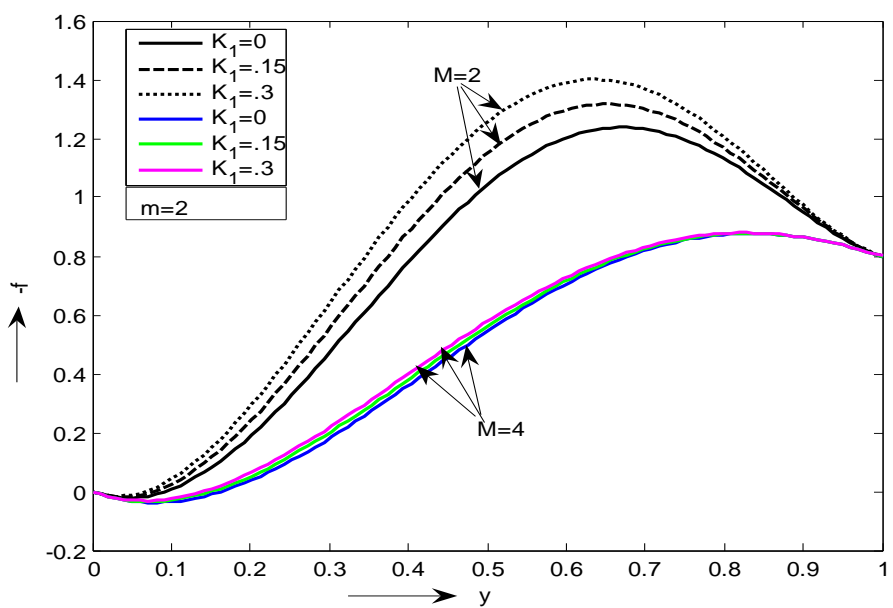

Figure 2: -f against y for $\mathrm{M}=2, \mathrm{M}=4, \mathrm{~m}=2, \alpha=.8, \beta=.8, \mathrm{R}_{\mathrm{e}}=.5, \omega \mathrm{t}=\pi / 4, \omega=5, \varepsilon=.01$

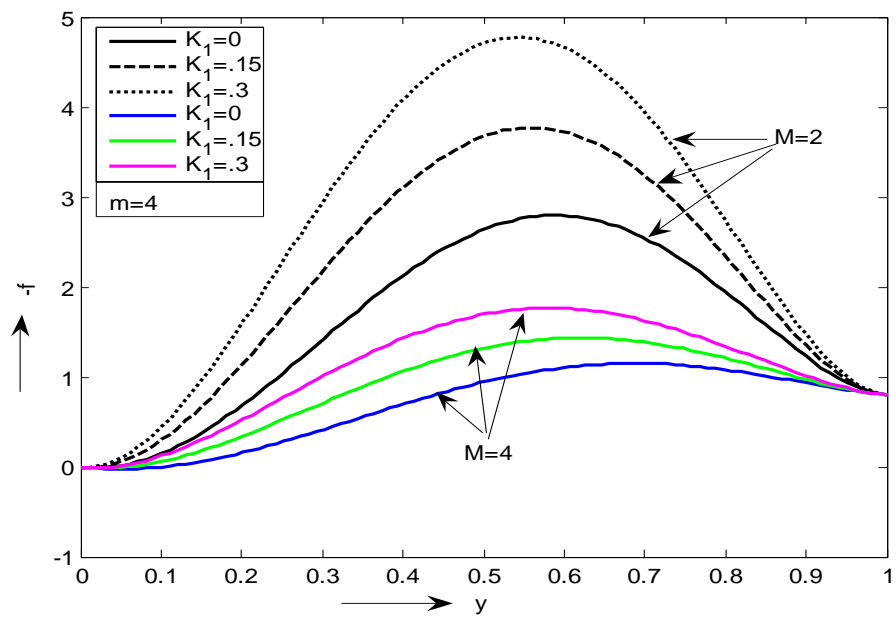

Figure 3: -f against y for $\mathrm{M}=2, \mathrm{M}=4, \mathrm{~m}=4, \alpha=.8, \beta=.8, \mathrm{R}_{\mathrm{e}}=.5, \omega \mathrm{t}=\pi / 4, \omega=5, \varepsilon=.01$

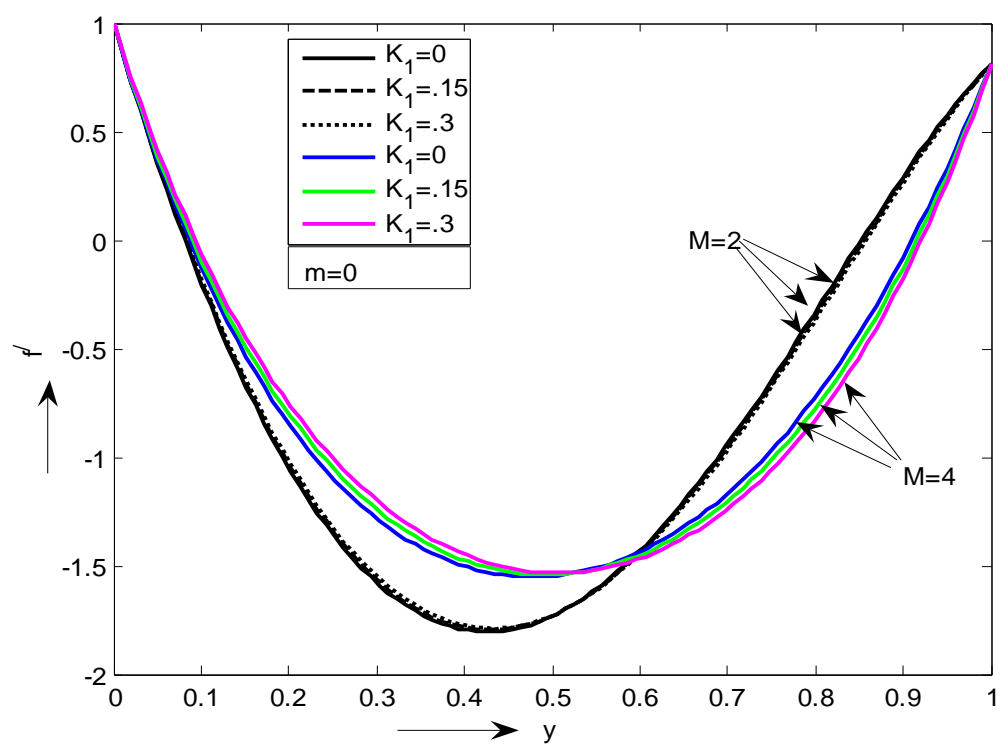

Figure 4: $\mathrm{f}^{\prime}$ against $\mathrm{y}$ for $\mathrm{M}=2, \mathrm{M}=4, \mathrm{~m}=0, \alpha=.8, \beta=.8, \mathrm{R}_{\mathrm{e}}=.5, \omega \mathrm{t}=\pi / 4, \omega=5, \varepsilon=.01$ 


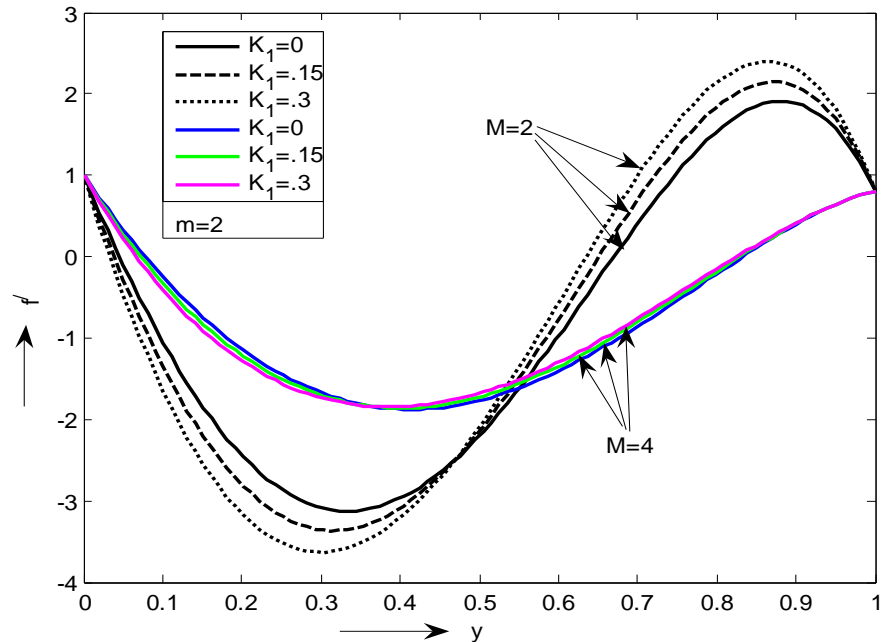

Figure 5: $\mathrm{f}^{\prime}$ against y for $\mathrm{M}=2, \mathrm{M}=4, \mathrm{~m}=2, \alpha=.8, \beta=.8, \mathrm{R}_{\mathrm{e}}=.5, \omega \mathrm{t}=\pi / 4, \omega=5, \varepsilon=.01$

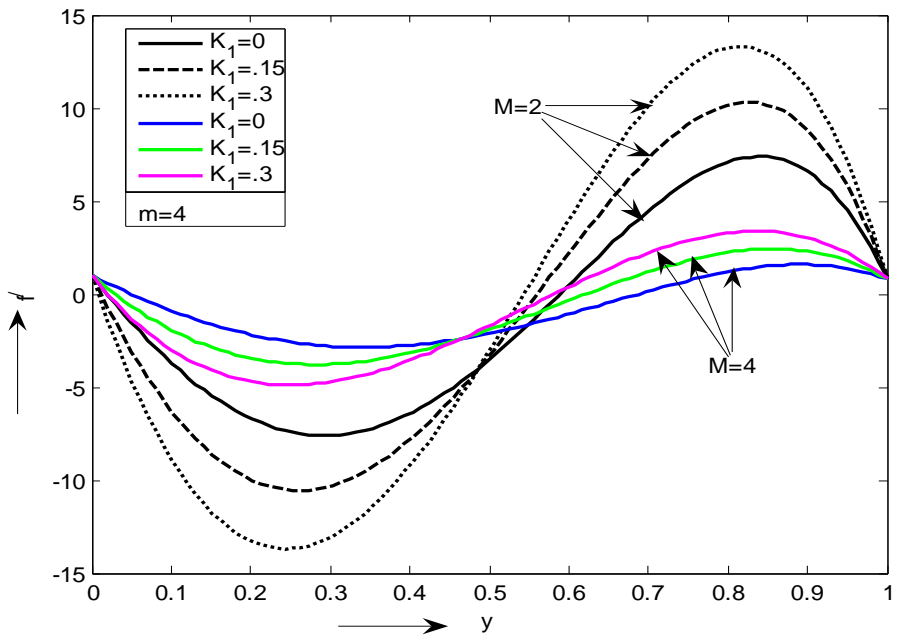

Figure 6: $\mathrm{f}^{\prime}$ against $\mathrm{y}$ for $\mathrm{M}=2, \mathrm{M}=4, \mathrm{~m}=4, \alpha=.8, \beta=.8, \mathrm{R}_{\mathrm{e}}=.5, \omega \mathrm{t}=\pi / 4, \omega=5, \varepsilon=.01$

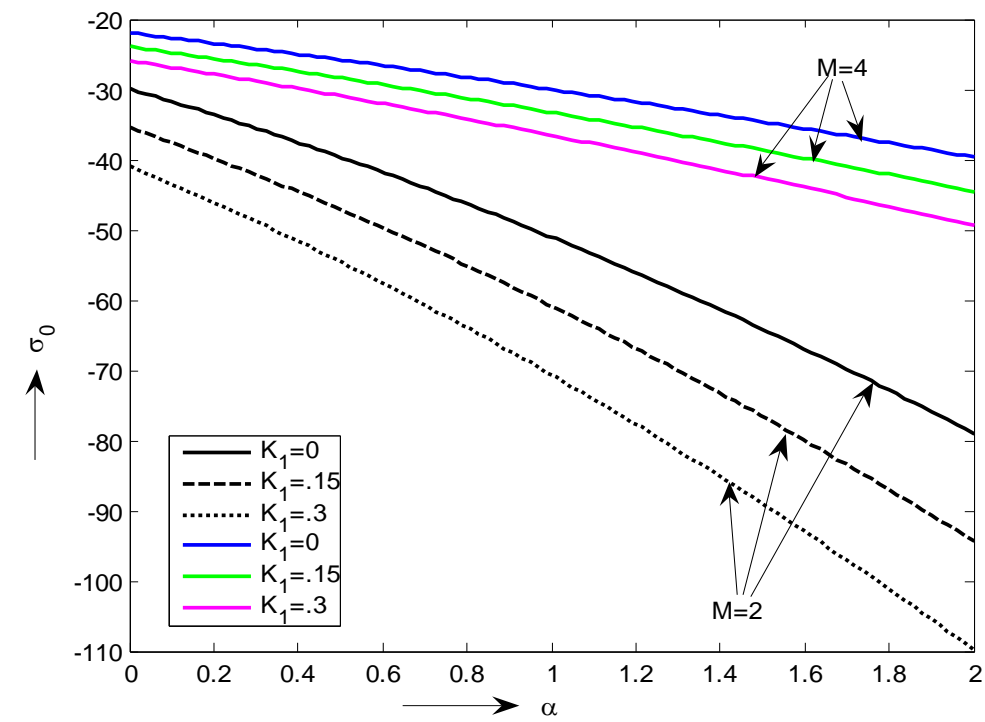

Fig-7: $\sigma_{0}$ against $\alpha$ on the plate $\mathrm{y}=0$ for $\mathrm{M}=2, \mathrm{M}=4, \mathrm{~m}=2, \beta=.8, \mathrm{Re}=.5, \omega \mathrm{t}=\pi / 4, \omega=5, \varepsilon=.01$ 


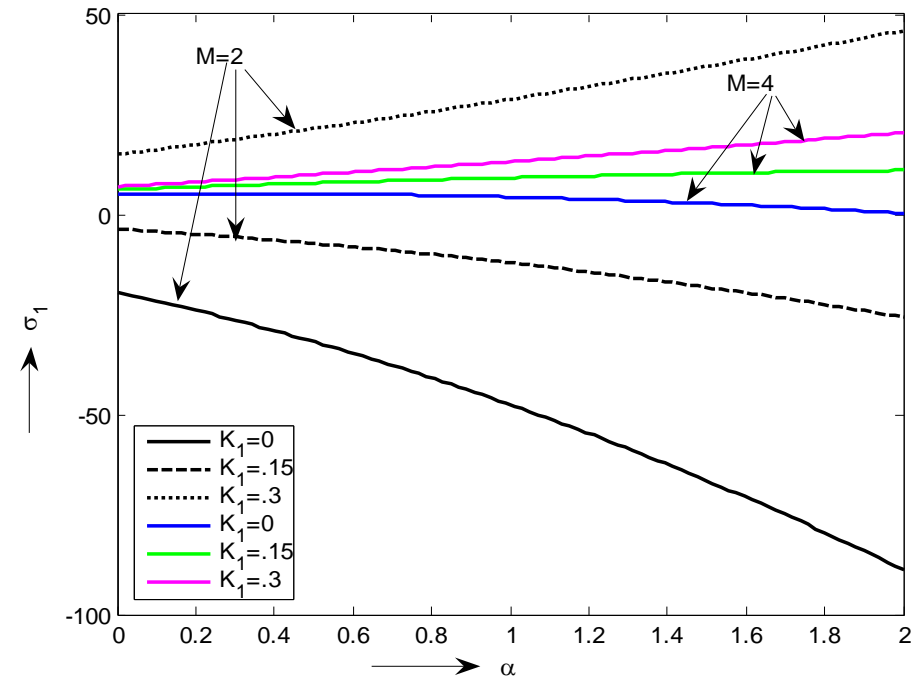

Fig- 8 : $\sigma_{1}$ against $\alpha$ on the plate $\mathrm{y}=1$ for $\mathrm{M}=2, \mathrm{M}=4, \mathrm{~m}=2, \beta=.8, \mathrm{Re}=.5, \omega \mathrm{t}=\pi / 4, \omega=5, \varepsilon=.01$

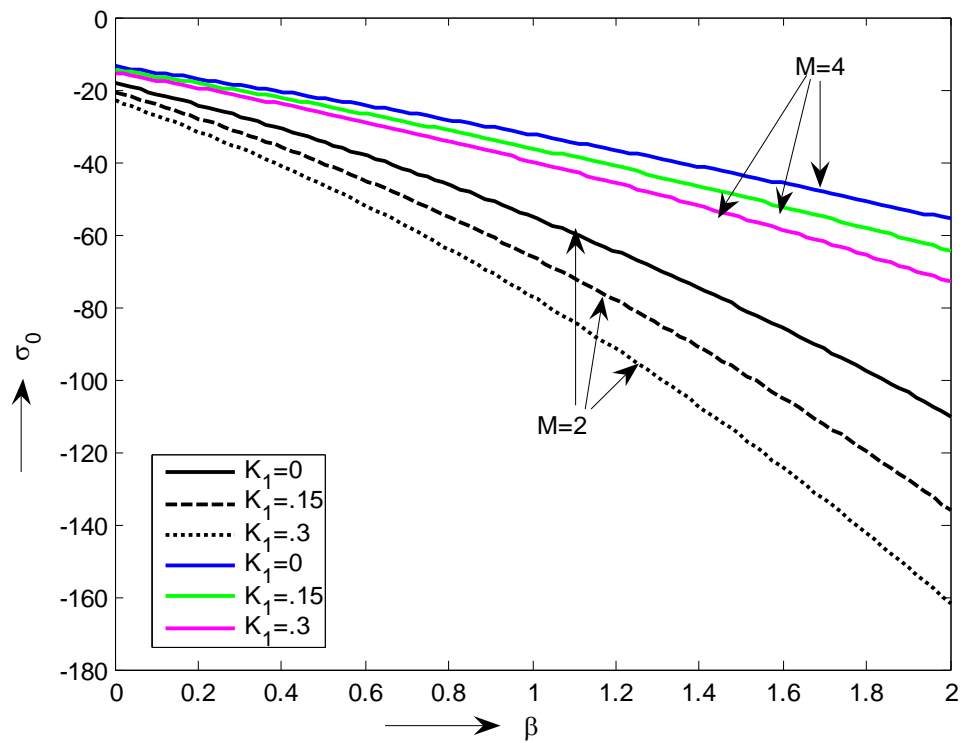

Fig-9: $\sigma_{0}$ against $\beta$ on the plate $\mathrm{y}=0$ for $\mathrm{M}=2, \mathrm{M}=4, \mathrm{~m}=2, \alpha=.8, \mathrm{Re}=.5, \omega \mathrm{t}=\pi / 4, \omega=5, \varepsilon=.01$

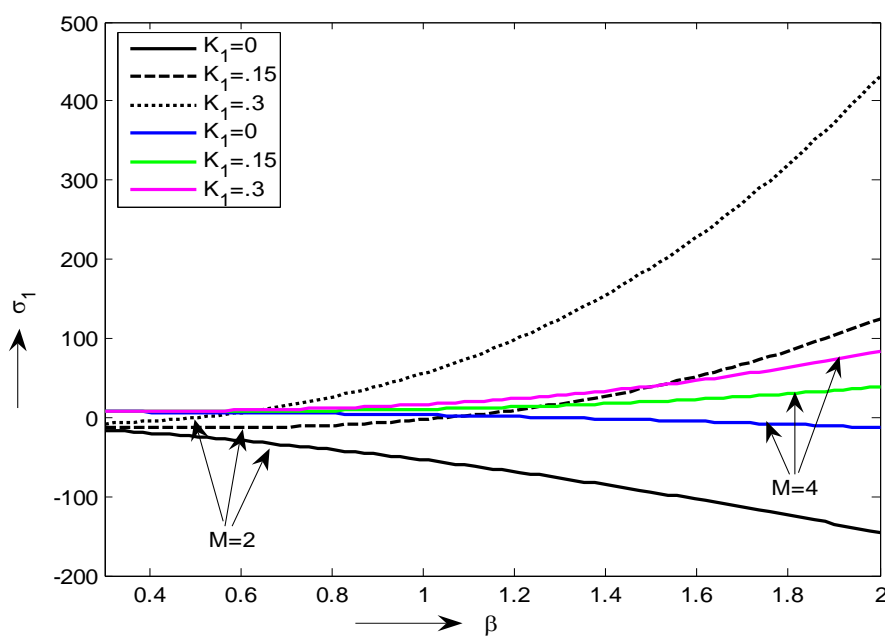

Fig-10: $\sigma_{1}$ against $\beta$ on the plate $\mathrm{y}=1$ for $\mathrm{M}=2, \mathrm{M}=4, \mathrm{~m}=2, \alpha=.8, \mathrm{Re}=.5, \omega \mathrm{t}=\pi / 4, \omega=5, \varepsilon=.01$ V. Conclusion 
An unsteady MHD flow of an electrically conducting visco-elastic fluid between two horizontal parallel non conducting plates in presence of a transverse magnetic field and the effects of Hall current where the lower plate is a stretching sheet while the upper one is a porous plate, which is oscillating in its own plane is studied for different values of visco-elastic parameter $\mathrm{K}_{1}$ in combination of other flow parameters.

From this study, we make the following conclusions:

- The flow field is considerably affected by the visco-elastic parameter.

- The velocity along $y$-direction diminishes in absence of Hall current effect but enhances in presence of Hall current effect with the growth of the visco-elastic parameter.

- The magnitude of the velocity along $y$-direction decelerates with the rising trend of magnetic parameter.

- The visco-elastic effect in velocity along $y$-direction is more prominent in the centre of the channel.

- The velocity along the stretching sheet enhances near the stretching sheet but diminishes near the moving sheet with the growth of the visco-elastic parameter in absence of Hall current effect.

- The velocity along the stretching sheet diminishes near the stretching sheet but enhances near the moving sheet with the growth of the visco-elastic parameter in presence of Hall current effect.

- The visco-elastic effect in velocity is prominent for variation of magnetic parameter in presence or absence of the Hall current effect.

- The skin-friction coefficient diminishes at the stretching sheet and enhances at the moving sheet with the growth of the visco-elastic parameter.

\section{References}

[1] A. K. Borkakoti, A.Bharali, Hydro Magnetic Flow and Heat Transfer between Two Horizontal, the Lower Plate Being a Stretching Sheet, Quart. Appl. Math, 40 (4), 1982, 461-467.

[2] T. C. Chiam, Micro Polar Fluid Flow over a Stretching Sheet, ZAMM, 62 (10), 1982, 565-568.

[3] K. R Rajagopal, Y. T. Na, A. S. Gupta, Flow of a Viscoelastic Fluid over Stretching Sheet, Rheol. Avta, 23 (2), 1984, 213-215

[4] R. S. Agarwal, R. Bhargava, A.V. S. Balaji, Finite Element Solution of Flow and Heat Transfer of a Micro Polar Fluid over a Stretching Sheet, Int. J. Engg. Sci., 27 (11), 1989, 1421-1428.

[5] H. I. Anderson, MHD Flow of Viscoelastic Vluid Past a Stretching Surface, Acta Mech., 95 (1-4), 1992, 227-230

[6] H. I. Anderson, K. H. Bech, B. S. Dandapat, Magnetohydrodynamics Flow of a Power Law Fluid over a Stretching Sheet, Int. J. Non-Linear Mechanics, 27 (6), 1992, 929-936.

[7] M. I. Char, Heat and Mass Transfer in a Hydromagnetic Flow of the Visco-elastic Fluid over a Stretching Sheet, J. Math, Anal. Appl., 186, 1994, 647-689.

[8] D. S. Chaun, Coupled Stretching Flow through a Channel Bounded by a Naturally Permeable Bed, Modeling, Measu. And Control ASME Press, 47 (4), 1993, 55-64.

[9] H.S. Takhar, G. Nath, Unsteady Flow over a Stretching Surface with a Magnetic Field in a Rotating Fluid, ZAMP, 49 (6), 1998, 989-1001.

[10] M. Kumari, H. S. Takhar, G. Nath, Analytical Solution of Boundary Layer Equation over a Stretching Sheet with Mass Transfer, Proc. Nat. Acad. Sci. India, 69A III (1999), 355- 372.

[11] P. R. Sharma, M. Mishra, Steady MHD Flow through a Horizontal Channel Lower Being a Stretching Sheet and Upper Being Permeable Plate Bounded by Porous Medium, Bull. Pure.Appl. Sci,. India, 20E (1), 2001, 175-181.

[12] D. K, Phukan, Hydromagnetic Flow and Heat Transfer over a Surface Stretching with a Power Law Velocity Distribution, Ganita, 54 (2), 2003, 177.

[13] S. Bhardwaj, Flow of a Fluid Due to Plane Stretching of a Sheet in a Porous Medium, Raj. Acad. Phyl. Sci., 3, 2004, 221-229.

[14] A. Lodtha, S. S. Tak, Heat Transfer with Viscos Dissipation in Hydromagnetic Flow over a Stretching Sheet, Ganita Sandesh, 19 (2), 2005, 209-215.

[15] A. S. Gupta, Hydromagnetic Flow Pas a Porous Plate with Hall Effects, Actamechanica, 22 (3-4), $1975,281-287$.

[16] P. Kumar, G. J. Singh, R.Lal, Thermal Instability of Walters B' Viscoelastic Fluid Permeated with Suspended Particles in Hydromagnetics in Porous Medium, Thermal Science, 8 (1), 2004, 51-61.

[17] P. Kumar, M. Singh, Instability of Two Rotating Viscoelastic (Walters B') Superposed Fluids with Suspended Particles in Porous Medium. Thermal Science, 11 (1), 2007, 93-102.

[18] M. Mostafa, , El-Din Salah, Laminar Fully Developed Mixed Convection with Viscous Dissipation in a UniformlyHeated Vertical Double-Passage Channel, Thermal Science, 11 (1), 2007, 27-41.

[19] A. Y. Bakier, M. A. Mansour. Combined of Magnetic Field and Thermophoresis Particle Deposition in Free Convection Boundary Layer From a Vertical Plate Embedded in a Porous Medium, Thermal Science, 11 (1), 2007, 65-74.

[20] I. A. Abdallah, Analytic Solution of Heat and Mass Transfer over a Permeable Stretching Plate Affected by Chemical Reaction, Internal Heating, Dufour-Soret Effect and Hall Effect, Thermal Science, 13 (2), 2009, 183-197.

[21] M. C.Raju, , N. A. Reddy, S. V. K. Verma, Hall-Current effects on Unsteady MHD Flow Between Stretching Sheet and an Oscillating Porous Upper Parallel Plate with Constant Suction, Thermal Science, 15 (2), 2011, 527-536.

[22] K. Walters, The motion of an elastico-viscous liquid contained between co-axial cylinders (II), Quart. J. Mech. Appl. Math., 13, $1960,444-461$

[23] K. Walters, The solution of flow problems in the case of materials with memories, J. Mecanique, 1, 1962, 473-478. 